Formatted: Justify Low, Left-to-right

Formatted: Font: $10 \mathrm{pt}$, Bold,

Underline, Complex Script Font: 10 pt, Not Bold

Formatted: Font: $11 \mathrm{pt}$, Complex

Script Font: $11 \mathrm{pt}$

Formatted: Justify Low, Line spacing: single

Formatted: Footer distance from

edge: $2 \mathrm{~cm}$

Formatted: Font: $8 \mathrm{pt}$, Complex Script Font: $8 \mathrm{pt}$

Formatted: Left, Indent: Before: 0

$\mathrm{cm}$, Line spacing: single

\section{matted}

Formatted: Font: 2 pt, Complex Script

Font: 2 pt

Formatted: Left, Indent: Before: 0 $\mathrm{cm}$, Line spacing: single

Formatted: Font: 9 pt, Not Italic Complex Script Font: 9 pt, Not Italic, Superscript

\section{... matted}

Formatted: Left, Indent: Before: 0 $\mathrm{cm}$, First line: $0 \mathrm{~cm}$, Line spacing:

single

\begin{tabular}{|l|l|}
\hline$\ldots$ matted \\
\hline$\ldots$ matted \\
\hline$\ldots$ matted \\
\hline$\ldots$ matted \\
\hline$\ldots$ matted \\
\hline$\ldots$ matted \\
\hline$\ldots$ matted \\
\hline$\ldots$ matted \\
\hline Formatted Table \\
\hline Formatted: Justify Low \\
\hline$\ldots$ matted \\
\hline$\ldots$ matted \\
\hline Formatted: Indent: First line: $0 \mathrm{~cm}$ \\
\hline$\ldots$ matted \\
\hline$\ldots$ matted \\
\hline$\ldots$ matted \\
\hline Formatted: Line spacing: single \\
\hline$\ldots$ matted \\
\hline$\ldots$ matted \\
\hline$\ldots$ matted \\
\hline
\end{tabular}

\begin{tabular}{|l|l|}
\hline$\ldots$ matted \\
\hline$\ldots$ matted \\
\hline Formatted: Left-to-right \\
\hline$\ldots$ matted \\
\hline
\end{tabular}

$\underline{\text { Assiut Vet. Med. J. Vol. } 58 \text { No. } 135 \text { October } 2012}$

\title{
IINFLUENCE OF CAMEL BREEDS AND AGES ON THE REPROD PERFORMANCE OF DROMEDARY CAMELS IN SAUDI ARABIA
}

$\rightarrow$ MOHAMMED, K.M.E. ${ }_{-}^{+*}$ and AL-MUTAIRI, S.E.

$\Rightarrow$ Animat ${ }^{*}$ Animal Reproduction Research Institute (ARRI), Giza, AL-Haram, Egypt, P.O. 12556

Camel Research Center, Sakaka/Al Jouf, Saudi Arabia, P.O.322

Correspondence email: kamel14@ hotmail.com

\section{ABSTRACT}

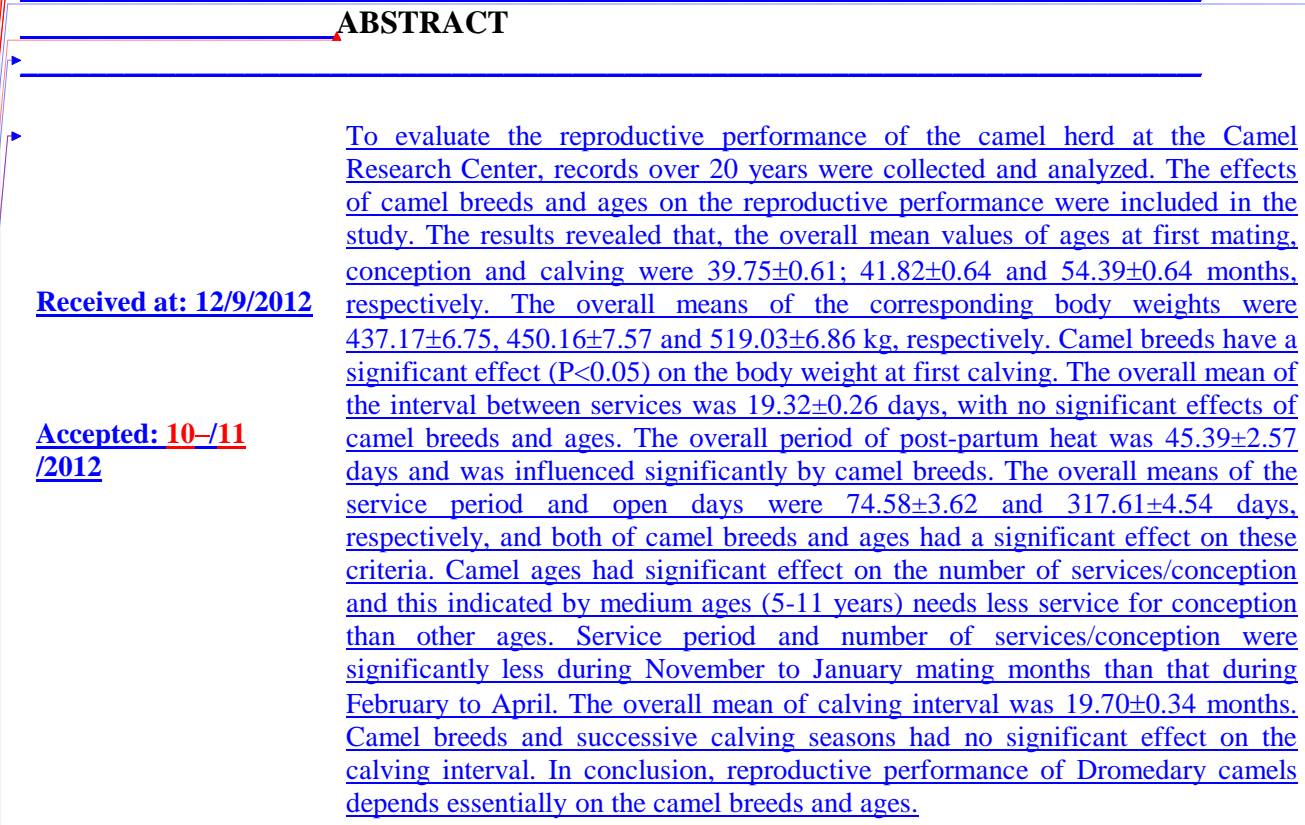

Key words: Dromedary camels, -reproductive performance, camel bree, $\rightarrow$

$\rightarrow$

To evaluate the reproductive performance of the camel herd at the Camel Research Center, records over 20 years were collected and analyzed. The effects of camel breeds and ages on the reproductive performance were included in the study. The results revealed that, the overall mean values of ages at first mating, conception and calving were $39.75 \pm 0.61 ; 41.82 \pm 0.64$ and $54.39 \pm 0.64$ months, respectively. The overall means of the corresponding body weights were $437.17 \pm 6.75,450.16 \pm 7.57$ and $519.03 \pm 6.86 \mathrm{~kg}$, respectively. Camel breeds have a significant effect $(\mathrm{P}<0.05)$ on the body weight at first ealving. The overall mean of the interval between services was $19.32 \pm 0.26$ days, with no significant effects of camel breeds and ages. The overall period of post-partum heat was $\rightarrow 45.39 \pm 2.57$ days and was influenced significantly by camel breeds. The overall means of the service period and open days were $7<$ and $317.61 \pm 4.54$ days, respectively, an eamel breeds and ages had a significant these criteria. Camel ages had signific: on the number of services/conception indicated by medium ages (5 11 yea less service for conception than otl Service period and numb servicesteonception were significan during November to January mating me that during February to April. The ove of calving interval was $19.70 \pm 0.34$ Camel breeds and successive calving se no significant effect on the calving in eonclusion, reproductive performe Dromedary camels depends essentiall eamel breeds and ages.

Key words: Dromedary can reproductive performance, camel br 


\begin{tabular}{|c|}
\hline $\begin{array}{l}\text { Formatted: Font: } 10 \mathrm{pt} \text {, Complex } \\
\text { Script Font: } 14 \mathrm{pt}\end{array}$ \\
\hline $\begin{array}{l}\text { Formatted: Justify Low, Left-to-right, } \\
\text { After: } 0 \mathrm{~cm}\end{array}$ \\
\hline Formatted: Left-to-right \\
\hline $\begin{array}{l}\text { Formatted: Justify Low, Left-to-right, } \\
\text { After: } 0 \mathrm{~cm}\end{array}$ \\
\hline $\begin{array}{l}\text { Formatted: Font: } 8 \text { pt, Bold, } \\
\text { Underline, Complex Script Font: } 8 \text { pt, } \\
\text { Not Bold, (Complex) Arabic (Egypt) }\end{array}$ \\
\hline $\begin{array}{l}\text { Formatted: Justify Low, Left-to-right, } \\
\text { After: } 0 \mathrm{~cm}\end{array}$ \\
\hline $\begin{array}{l}\text { Formatted: Indent: First line: } 0 \mathrm{~cm} \text {, } \\
\text { Line spacing: single }\end{array}$ \\
\hline $\begin{array}{l}\text { Formatted: Font: } 10 \mathrm{pt} \text {, Complex } \\
\text { Script Font: } 10 \mathrm{pt}\end{array}$ \\
\hline Formatted: Line spacing: single \\
\hline $\begin{array}{l}\text { Formatted: Justify Low, Indent: First } \\
\text { line: } 0.95 \mathrm{~cm} \text {, Line spacing: single }\end{array}$ \\
\hline $\begin{array}{l}\text { Formatted: Font: } 10 \mathrm{pt} \text {, Complex } \\
\text { Script Font: } 10 \mathrm{pt}\end{array}$ \\
\hline ... matted \\
\hline ... matted \\
\hline ... matted \\
\hline ... matted \\
\hline ... matted \\
\hline ... matted \\
\hline ... matted \\
\hline ... matted \\
\hline ... matted \\
\hline ... matted \\
\hline ... matted \\
\hline
\end{tabular}

\begin{tabular}{|c|}
\hline ... matted \\
\hline ... matted \\
\hline ... matted \\
\hline$\ldots$ matted \\
\hline$\ldots$ matted \\
\hline ... matted \\
\hline ... matted \\
\hline$\ldots$ matted \\
\hline ... matted \\
\hline ... matted \\
\hline ... matted \\
\hline ... matted \\
\hline ... matted \\
\hline ... matted \\
\hline Formatted: Left-to-right \\
\hline ... matted \\
\hline
\end{tabular}
A

\section{INTRODUCTION}

The one-humped camel is a unique producer of food in the arid and semi-arid zones of the world. It is the only animal that can exist for several weeks without water and still able to provide its offspring and humans with milk (Yagil, 1982). The total -population of camels in the world was estimated to be $\rightarrow$ about 19 million, 14 million $(73 \%)$ of which are in - Africa and the Near East and 5 million (27\%) in Asia (FAO 1992). The total population of dromedary is estimated to be around 1.6 million camels within the Arabian Peninsula, about 53\% found in Saudi Arabia (FAO statistics, 2011). However, there is an important gap between the official number of camel heads according to FAO statistics (280,000 in 2009) and the national estimation published by the Ministry of Agriculture in Saudi Arabia (830,000 heads). Based on this last estimation, the camel population is $51 \%$ of the total tropical livestock in the country (Abdallahl and Bernard Faye, 2012). The importance of camel in Saudi Arabia is clearly underlined by these data. The total population of dromedary in the Arabian Peninsula was estimated at approximately 1.6 million camels, about 53\% in Saudi Arabia (Al-Eknah, 2008), Camels in Saudi Arabia play a major role in supplying the desert indwellers with milk and meat under extremely hostile conditions of temperature, drought and lack of pasture (Yagil and Etzion, 1980). Camel breeds derive their names from the areas of their habitats in dry and hot zones (North Africa, the North East and West Central Asia) as well as according to the tribe name (Wilson, 1984). A recent classification of camel breeds based on their economical traits was suggested by Wardeh (1989). Accordingly, Camels are assigned to four main classes: beef, dairy, dual purpose and racing camels. However, Saoud et al. (1988) and Basmaeil, et al--. (2012) identified native camel breeds in the Saudi Arabia according to coat colors and production into Magahiem (black color, best milking breed); Maghatier (white color, moderate in milk production) Safrah (color mixture between the white and red) and Hamrah (color overlap between red and blond, more suitable for meat production than milk).- The breeding of camels is at high risk because of the slow reproduction rate (Dahl and Hjort, 1979). Full reproductive capacity of the female camel-is reached at 6 years (Singh, 1966; Khetami, 1970), but it can be bred at 3-5 years of age (Matharu, 1966; Williamson and Payne, 1978). Yasin and Wahid (1957) reported that the female camel would breed until 30 years of age.

$\rightarrow$ The production and reproduction in camels are affected by many factors such as late puberty, restricted breeding season, induced ovulation, long gestation period and long calving interval. These factors may constitute a major reason for the long generation interval in camels. The investigation aimed to study the re performance of Saudi Arabian camels unde management system will shed more lig influence of -age and camel breeds reproductive performance traits.

\section{MATERIALS and METHI}

The used records used-were collected fron of the camel station at the Camel Research Sakaka, Al Jouf in the Nnortheastern par Arabia. These records were used to ev reproductive performance through measu the fertility parameters of Arabian car intensive controlled management. Theebservations of reproductive parameters $\mathrm{wl}$ inincluded in this study included, data of $a$ female camel whichere collected from sexually mature camels during consecutiv seasons over a period of 20 years (1985 to : animals were fed the available hay ad-libidu $\mathrm{kg}$ concentrate pellets (containing 16\% cru 2.5-3.0\% crude fat, $4.9-8.5 \%$ crude fibe $0.69-0.7 \%$, phosphorus $0.4 \%$ and salt $1 \%$ breeding season variable amounts of alt offered to the animals. Supplementary feec form of whole dates and bran were als irregularly.

The studied reproductive performance in following traits which described by EL-A (1997): (1) Ages (months) and weights (k mating, first conception and first calving, ( between services (days) within the same season which annually starts at early Noven April and may extended to early May, (3) $\mathrm{P}_{\mathrm{r}}$ period (days); period between calving to ol first heat, (4) Service period (days); perioc from calving to successful conception withi: breeding season, (5) Open days period (day from calving to last successful service whic] to forthcoming breeding season, (6) $\mathrm{N}$ services/conception, (7) Calving interv: period between two consecutive calving's-.

The influence of camel breeds and ages on 1 were studied. Đata $\underline{\text { O}}$ ebtained data acc available observations of reproductive $t_{1}$ tabulated into 2 fixed factors (camel breeds and statistically analyzed using the SPSS ve Windows statistical software package. The also subjected to analysis of variance usir Linear Model (GLM) procedure and multiple comparisons for observed $r$ Duncan's test for performances in relati suggested factors.

\section{RESULTS}




\begin{tabular}{|c|}
\hline $\begin{array}{l}\text { Formatted: Font: } 10 \mathrm{pt} \text {, Complex } \\
\text { Script Font: } 14 \mathrm{pt}\end{array}$ \\
\hline $\begin{array}{l}\text { Formatted: Justify Low, Left-to-right, } \\
\text { After: } 0 \mathrm{~cm}\end{array}$ \\
\hline Formatted: Left-to-right \\
\hline ... matted \\
\hline ... matted \\
\hline ... matted \\
\hline ... matted \\
\hline ... matted \\
\hline ... matted \\
\hline ... matted \\
\hline ... matted \\
\hline ... matted \\
\hline ... matted \\
\hline ... matted \\
\hline ... matted \\
\hline ... matted \\
\hline ... matted \\
\hline ... matted \\
\hline ... matted \\
\hline ... matted \\
\hline ... matted \\
\hline ... matted \\
\hline ... matted \\
\hline ... matted \\
\hline Formatted: Indent: Before: $0 \mathrm{~cm}$ \\
\hline ... matted \\
\hline Formatted Table \\
\hline Formatted: Centered \\
\hline Formatted: Centered \\
\hline Formatted: Centered \\
\hline Formatted: Centered \\
\hline Formatted: Centered \\
\hline Formatted: Centered \\
\hline ... matted \\
\hline ... matted \\
\hline Formatted: Indent: Before: $0 \mathrm{~cm}$ \\
\hline ... matted \\
\hline Formatted Table \\
\hline Formatted: Centered \\
\hline Formatted: Centered \\
\hline ... matted \\
\hline Formatted: Left-to-right \\
\hline ... matted \\
\hline
\end{tabular}

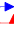

2

$\rightarrow$ Assiut Vet. Med. J. Vol. 58 No. 135 October 2012

-As shown in Tables 1 and 2, the overall mean values of ages at first mating, conception and calving were $39.75+0.61 ; 41.82+0.64$ and $54.39+0.64$ months, respectively. The overall means of the corresponding body weights were $437.17 \pm 6.75,450.16 \pm 7.57$ and $519.03+6.86 \mathrm{~kg}$, respectively. The results indicated that, the differences between the camels' breeds (Magahiem, Maghatier, Hamrah and Safrah) were not significant for the studied ages and body weights at first services and conception. However, Safrah camel breed had lesser $(\mathrm{P}<0.05)$ body weight $(499.00 \pm 14.04$ $\mathrm{Kg}$ ) at first calving than other camel breeds.

The overall mean values of the interval between services were $19.72 \pm 0.51,19.13 \pm 0.42,19.56 \pm 0.78$ and $19.50 \pm 0.50$ days for Magahiem, Maghatier, Safah and Hamrah camel breeds, respectively, whereas, the mean values of the same criteria for camel ages $\leq 5,5$ $7,7-9,9-11$ and $\geq 11$ years were $19.28 \pm 0.32$, $18.78 \pm 0.63,20.41 \pm 0.84,19.34 \pm 0.84$ and $18.93 \pm 0.90$ days, respectively (Table 3 ). Camel breeds and ages didn't have a non-significant effect on this trait.

The effect of camel breeds and ages on post-partum period are illustrated in Ttable (4). Results indicated that, Hamrah camel breed had significant $(\mathrm{P}<0.05)$ shorter period ( $36.83 \pm 3.73$ days) than other breeds. However, no significant effect to camel ages on the post-partum period, and the overall period was $45.39 \pm 2.57$ days.

The influences of camel breeds and ages on the service periods are illustrated in Ttable (5)5. Results indicated that, Hamrah camel breed had significant $(\mathrm{P}<0.05)$ longer period $(93.21 \pm 7.42$ days $)$ than other breeds, at the same time, female camel ages $\rightarrow 11$

years old had significant $(\mathrm{P}<0.05)$ lonє $(90.88 \pm 10.85$ days) than other studied ages.

The effects of camel breeds and ages on the period are showed in Table (6). The open $\mathrm{d}$ were affected by camel breeds and agt Maghatier camel breed recorded longer period (329.51 \pm 7.50 days) than other breec camel ages 5-7 years reported longer $(\mathrm{P}<0 . \mathrm{C}$ $(342.46 \pm 7.59$ days) than other studied influences of camel breeds and ages on $t$ service/conception are illustrated in Ttabls overall mean value of number of services/، was $2.57 \pm 0.02$ services. Results indicated th camels ages had significant effect $(\mathrm{P}<0 . \mathrm{C}$ number of service/conception and this in medium ages (7 to 9 years) needs less st conception $(2.05 \pm 0.20)$, on the other han $(\leq 5$ years $)$ and older ages $(\geq 11$ years $) \mathrm{n}$ services for conception ( $2.88 \pm 0.17$ and respectively)-(Table 7). However, the -car had no significant effect on this trait.

Measurement of calving interval is an imp herd performance monitoring tool. As d Ttable (8), the overall mean of calving in $19.70+0.34$ months; and no significant diff the calving interval between camel b1 successive calving seasons. However, c: show substantial difference in the calving $\mathrm{i}$ she-camels age advanced the interval incr this phenomenon was denoted up to the 1 season (Table 8). Calving interval distribut she-camel population is shown in Fig. calving interval classes were $<15,15-17,18$ and $>24$ months and the corresponding $\mathrm{p}$ were $7.27 \%, 17.27 \%, 35.46 \%, 31.82 \%$ and the she-camels, respectively.

$\rightarrow$ Table 1: Influence of camel breeds on the ages at first service, conception and calving (Mean \pm SE)

\begin{tabular}{|c|c|c|c|c|}
\hline \multirow{2}{*}{$\begin{array}{l}\text { Camel } \\
\text { Breeds } \\
\end{array}$} & \multirow{2}{*}{ No. of observations } & \multicolumn{3}{|c|}{ Ages (Months) } \\
\hline & & $\underline{1}^{\text {st }}$ Service & $1^{\mathrm{st}}$ Conception & $\underline{1^{\text {st }} \text { Calving }}$ \\
\hline Magahiem & $\underline{38}$ & $\underline{41.21 \pm 1.34}$ & $\underline{42.90 \pm 1.41}$ & $\underline{55.53 \pm 1.41}$ \\
\hline Maghatier & $\underline{60}$ & $\underline{39.33 \pm 1.07}$ & $\underline{40.77 \pm 1.13}$ & $\underline{53.36 \pm 1.12}$ \\
\hline$\underline{\text { Safrah }}$ & $\underline{46}$ & $\underline{40.09 \pm 1.22}$ & $\underline{42.65 \pm 1.28}$ & $\underline{55.21 \pm 1.28}$ \\
\hline$\underline{\text { Hamrah }}$ & $\underline{44}$ & $\underline{38.36 \pm 1.25}$ & $\underline{40.96 \pm 1.31}$ & $\underline{53.48 \pm 1.31}$ \\
\hline$\underline{\text { Overall }}$ & $\underline{188}$ & $\underline{39.75 \pm 0.61}$ & $\underline{41.82 \pm 0.64}$ & $\underline{54.39 \pm 0.64}$ \\
\hline
\end{tabular}

Table 2: Influence of camel breeds on the body weight at first service, conception and calving (Mean \pm

\begin{tabular}{|c|c|c|c|c|}
\hline \multirow[b]{2}{*}{$\underline{\text { Camel Breeds }}$} & \multirow{2}{*}{ No. of observations } & \multicolumn{3}{|c|}{ Body weights (kg) } \\
\hline & & $1^{\text {st }}$ Service & $1^{\text {st }}$ Conception & $1^{\text {st }}$ Calving \\
\hline Magahiem & $\underline{14}$ & $\underline{442.57 \pm 15.66}$ & $456.29 \pm 17.58$ & $\underline{504.57 \pm 15.92^{\mathrm{ab}}}$ \\
\hline
\end{tabular}




\begin{tabular}{|c|}
\hline ... matted \\
\hline ... matted \\
\hline ... matted \\
\hline ... matted \\
\hline ... matted \\
\hline$\ldots$ matted \\
\hline ... matted \\
\hline ... matted \\
\hline ... matted \\
\hline ... matted \\
\hline ... matted \\
\hline ... matted \\
\hline ... matted \\
\hline ... matted \\
\hline ... matted \\
\hline ... matted \\
\hline ... matted \\
\hline ... matted \\
\hline ... matted Table \\
\hline ... matted \\
\hline ... matted \\
\hline ... matted \\
\hline ... matted \\
\hline ... matted \\
\hline ... matted \\
\hline ... matted \\
\hline ... matted \\
\hline ... matted \\
\hline ... matted \\
\hline ... matted \\
\hline ... matted \\
\hline ... matted \\
\hline ... matted \\
\hline ... matted \\
\hline ... matted \\
\hline ... matted \\
\hline ... matted Table \\
\hline ... matted \\
\hline ... matted \\
\hline ... matted \\
\hline ... matted \\
\hline ... matted \\
\hline ... matted \\
\hline ... matted \\
\hline ... matted \\
\hline ... matted \\
\hline ... matted \\
\hline ... matted \\
\hline ... matted \\
\hline ... matted Table \\
\hline ... matted \\
\hline ... matted \\
\hline ... matted \\
\hline ... matted \\
\hline ... matted \\
\hline ... matted \\
\hline
\end{tabular}

$\rightarrow$ Assiut Vet. Med. J. Vol. 58 No. 135 October 2012

$\rightarrow$

$\begin{array}{lllll}\text { Maghatier } & \underline{44} & \underline{444.77 \pm 8.84} & \underline{460.41 \pm 9.91} & \underline{530.05 \pm 8.98^{\mathrm{ab}}}\end{array}$

$\begin{array}{lllll}\underline{\text { Safrah }} \quad \underline{18} & \underline{439.44 \pm 13.81} & \underline{445.56 \pm 15.50} & \underline{499.00 \pm 14.04^{\mathrm{b}}}\end{array}$

\begin{tabular}{lllll}
$\underline{\text { Hamrah }}$ & $\underline{16}$ & $\underline{421.88 \pm 14.65}$ & $\underline{438.38 \pm 16.44}$ & $\underline{542.50 \pm 14.89^{\mathrm{a}}}$ \\
\hline
\end{tabular}

$\rightarrow$\begin{tabular}{lllll} 
Overall & $\underline{92}$ & $\underline{437.17 \pm 6.75}$ & $\underline{450.16 \pm 7.57}$ & $\underline{519.03 \pm 6.86}$ \\
\hline
\end{tabular}

$\rightarrow$ Means in the same column with different superscripts differ significantly $(\mathrm{P}<0.05)$.

Table 3: Influence of camel breeds and ages on the interval between services (Mean $\pm \mathrm{SE}$ )

\begin{tabular}{|c|c|c|c|c|c|c|c|}
\hline \multirow{2}{*}{$\vec{r}$} & \multirow{2}{*}{$\underline{\text { Ages }}$} & \multirow{2}{*}{$\underline{\text { No. of observations }}$} & \multicolumn{4}{|c|}{$\begin{array}{c}\text { Camel Breeds } \\
\text { (Number of observations) }\end{array}$} & \multirow{2}{*}{$\underline{\underline{\text { Overall }}}$} \\
\hline & & & $\frac{\frac{\text { Magahiem }}{(\mathrm{n}=186)}}{\underline{4}}$ & $\begin{array}{l}\frac{\text { Maghatier }}{\underline{(\mathrm{n}=238)}} \\
\end{array}$ & $\underline{\underline{\text { Safrah }}} \underline{\underline{(\mathrm{n}=90)}}$ & $\underline{\underline{\text { Hamrah }}}$ & \\
\hline - & $\leq 5$ & $\underline{339}$ & $\underline{18.55 \pm 0.82}$ & $\underline{19.37 \pm 0.63}$ & $\underline{19.46 \pm 0.92}$ & $\underline{19.57 \pm 0.66}$ & $\underline{19.28 \pm 0.32}$ \\
\hline & $\underline{5-7}$ & $\underline{105}$ & $\underline{17.93 \pm 1.65}$ & $\underline{19.22 \pm 0.89}$ & $\underline{19.58 \pm 1.84}$ & $\underline{17.65 \pm 1.55}$ & $\underline{18.78 \pm 0.63}$ \\
\hline & $\underline{7-9}$ & $\underline{90}$ & $\underline{21.86 \pm 1.20}$ & $\underline{18.88 \pm 1.11}$ & $\underline{18.00 \pm 4.51}$ & $\underline{21.29 \pm 1.55}$ & $\underline{20.41 \pm 0.84}$ \\
\hline 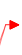 & $\underline{9-11}$ & $\underline{72}$ & $\underline{20.09 \pm 1.13}$ & $\underline{18.92 \pm 1.84}$ & $21.00 \pm 2.02$ & $\underline{16.62 \pm 1.77}$ & $\underline{19.34 \pm 0.84}$ \\
\hline & $\geqq 11$ & $\underline{81}$ & $\underline{20.61 \pm 1.20}$ & $\underline{18.17 \pm 1.50}$ & $\underline{18.20 \pm 2.85}$ & $\underline{17.45 \pm 1.43}$ & $\underline{18.93 \pm 0.90}$ \\
\hline$\rightarrow$ & Overall & $\underline{687}$ & $\underline{19.72 \pm 0.51}$ & $\underline{19.13 \pm 0.42}$ & $\underline{19.56 \pm 0.78}$ & $\underline{19.50 \pm 0.50}$ & $\underline{19.32 \pm 0.26}$ \\
\hline
\end{tabular}

Table 4: Influence of camel breeds and ages on the post-partum period (Mean \pm SE)

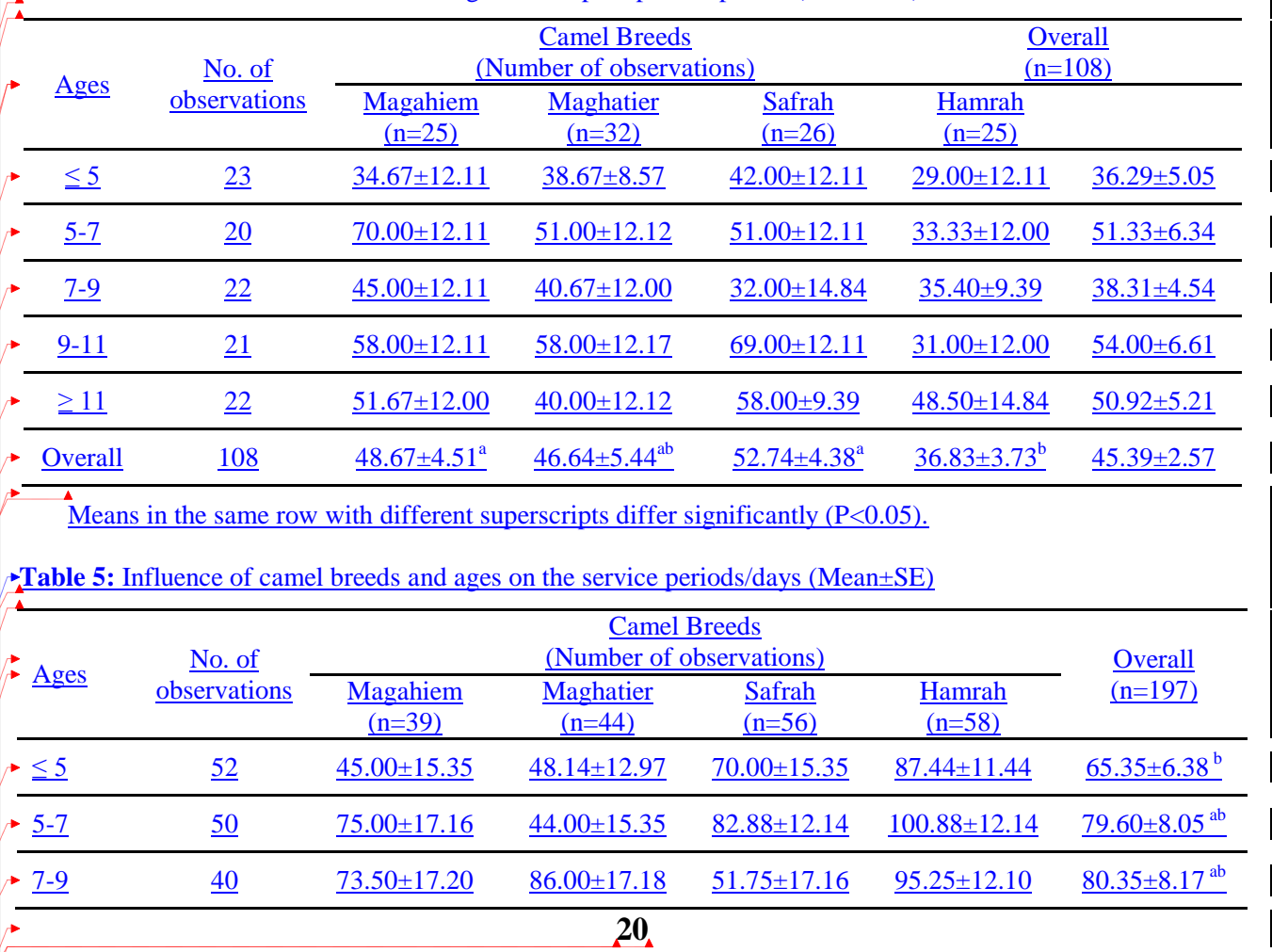




\begin{tabular}{|c|}
\hline ... matted \\
\hline ... matted \\
\hline ... matted \\
\hline ... matted \\
\hline ... matted \\
\hline ... matted \\
\hline ... matted \\
\hline ... matted \\
\hline ... matted \\
\hline ... matted \\
\hline ... matted \\
\hline ... matted \\
\hline ... matted \\
\hline ... matted \\
\hline ... matted \\
\hline ... matted \\
\hline ... matted \\
\hline ... matted \\
\hline ... matted \\
\hline ... matted \\
\hline ... matted \\
\hline ... matted \\
\hline ... matted \\
\hline ... matted \\
\hline ... matted \\
\hline ... matted \\
\hline ... matted \\
\hline ... matted \\
\hline ... matted \\
\hline ... matted \\
\hline ... matted \\
\hline ... matted \\
\hline ... matted \\
\hline ... matted \\
\hline ... matted \\
\hline ... matted \\
\hline ... matted Table \\
\hline ... matted \\
\hline ... matted \\
\hline ... matted \\
\hline ... matted \\
\hline ... matted \\
\hline ... matted \\
\hline ... matted \\
\hline ... matted \\
\hline ... matted \\
\hline ... matted \\
\hline ... matted Table \\
\hline ... matted \\
\hline ... matted \\
\hline ... matted \\
\hline ... matted \\
\hline ... matted \\
\hline
\end{tabular}

$\rightarrow$

$\rightarrow$ Assiut Vet. Med. J. Vol. 58 No. 135 October 2012

\begin{tabular}{lllllll}
$\rightarrow \underline{9-11}$ & $\underline{30}$ & $\underline{82.75 \pm 17.16}$ & $\underline{56.67 \pm 19.82}$ & $\underline{43.00 \pm 15.35}$ & $\underline{66.00 \pm 19.82}$ & $\underline{60.93 \pm 7.20^{\mathrm{b}}}$ \\
\hline$\underline{\underline{211}}$ & $\underline{25}$ & $\underline{82.40 \pm 21.71}$ & $\underline{113.67 \pm 19.82}$ & $\underline{73.33 \pm 14.01}$ & $\underline{149.00 \pm 34.33}$ & $\underline{90.88 \pm 10.85^{\mathrm{a}}}$ \\
\hline$\underline{\text { Overall }}$ & $\underline{197}$ & $\underline{69.54 \pm 7.41^{\mathrm{b}}}$ & $\underline{64.18 \pm 7.62^{\mathrm{b}}}$ & $\underline{66.96 \pm 5.80^{\mathrm{b}}}$ & $\underline{93.21 \pm 7.42^{\mathrm{a}}}$ & $\underline{74.58 \pm 3.62}$
\end{tabular}

Means in the same column with different superscripts differ significantly $(\mathrm{P}<0.05)$.

Means in the same row with different superscripts differ significantly $(\mathrm{P}<0.05)$.

Table 6: Influence of camel breeds and ages on the open days period (Mean \pm SE)

\begin{tabular}{|c|c|c|c|c|c|c|}
\hline \multirow{2}{*}{$\rightarrow$ Ages } & \multirow{2}{*}{$\begin{array}{l}\frac{\text { No. of }}{\text { observations }} \\
\underline{n^{\prime}}\end{array}$} & \multicolumn{4}{|c|}{$\begin{array}{c}\text { Camel Breeds } \\
\text { (Number of observations) }\end{array}$} & \multirow{2}{*}{$\frac{\text { Overall }}{(\mathrm{n}=272)}$} \\
\hline & & $\frac{\text { Magahiem }}{(\mathrm{n}=46)}$ & $\frac{\text { Maghatier }}{(\mathrm{n}=81)}$ & $\begin{array}{l}\text { Safrah } \\
(\mathrm{n}=63)\end{array}$ & $\underline{\text { Hamrah }}$ & \\
\hline$\rightarrow \leqq 5$ & $\underline{78}$ & $\underline{300.71 \pm 17.32}$ & $\underline{345.88 \pm 12.96}$ & $\underline{343.54 \pm 13.23}$ & $\underline{282.60 \pm 16.73}$ & $\underline{324.89 \pm 7.28^{\mathrm{bc}}}$ \\
\hline$\rightarrow 5-7$ & $\underline{55}$ & $\underline{365.40 \pm 28.97}$ & $\underline{356.40 \pm 14.49}$ & $\underline{336.29 \pm 17.32}$ & $\underline{323.25 \pm 16.20}$ & $\underline{342.46 \pm 7.59^{\mathrm{c}}}$ \\
\hline$\rightarrow 7-9$ & $\underline{45}$ & $\underline{305.40 \pm 28.97}$ & $\underline{262.75 \pm 22.91}$ & $\underline{275.80 \pm 28.97}$ & $\underline{327.00 \pm 22.91}$ & $\underline{293.23 \pm 17.59^{\mathrm{ab}}}$ \\
\hline$\rightarrow 2-11$ & $\underline{44}$ & $\underline{297.50 \pm 32.39}$ & $\underline{301.40 \pm 28.97}$ & $\underline{315.86 \pm 24.49}$ & $\underline{227.67 \pm 21.60}$ & $\underline{278.28 \pm 16.62^{\mathrm{a}}}$ \\
\hline 11 & $\underline{50}$ & $\underline{282.80 \pm 28.97}$ & $\underline{317.65 \pm 13.51}$ & $\underline{299.92 \pm 17.97}$ & $\underline{327.22 \pm 21.59}$ & $\underline{311.28 \pm 8.43^{\mathrm{ab}}}$ \\
\hline Dverall & $\underline{272}$ & $308.12 \pm 12.20^{\mathrm{ab}}$ & $\underline{329.51 \pm 7.50^{\mathrm{b}}}$ & $324.48 \pm 7.30^{\text {ab }}$ & $297.82 \pm 8.63^{\mathrm{a}}$ & $\underline{317.61 \pm 4.54}$ \\
\hline
\end{tabular}

Means in the same column with different superscripts differ significantly $(\mathrm{P}<0.05)$.

Means in the same row with different superscripts differ significantly $(\mathrm{P}<0.05)$.

Table 7: Influence of camel breeds and ages on the number of services/conception (Mean \pm SE)

\begin{tabular}{|c|c|c|c|c|c|c|}
\hline \multirow{2}{*}{$\overrightarrow{\text { Ages }}$} & \multirow{2}{*}{$\begin{array}{l}\text { No. of } \\
\text { observations }\end{array}$} & \multicolumn{4}{|c|}{$\begin{array}{c}\text { Camel Breeds } \\
\text { (no. of observations) }\end{array}$} & \multirow[t]{2}{*}{$\begin{array}{l}\underline{\text { Overall }} \\
\underline{\mathrm{n}=319)} \\
\end{array}$} \\
\hline & & $\frac{\text { Magahiem }}{\underline{(\mathrm{n}=62)}}$ & $\frac{\text { Maghatier }}{(\mathrm{n}=87)}$ & 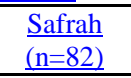 & 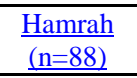 & \\
\hline$\rightarrow \leq 5$ & $\underline{121}$ & $\underline{2.92 \pm 0.48}$ & $\underline{2.76 \pm 0.26}$ & $\underline{2.37 \pm 0.33}$ & $\underline{3.39 \pm 0.29}$ & $2.88 \pm 0.17^{\mathrm{b}}$ \\
\hline$\rightarrow \underline{5-7}$ & $\underline{74}$ & $\underline{2.53 \pm 0.45}$ & $\underline{2.42 \pm 0.35}$ & $\underline{2.19 \pm 0.43}$ & $\underline{2.21 \pm 0.40}$ & $2.34 \pm 0.18^{a b}$ \\
\hline$\rightarrow \underline{7-9}$ & $\underline{43}$ & $\underline{2.44 \pm 0.58}$ & $\underline{2.36 \pm 0.46}$ & $1.13 \pm 0.61$ & $\underline{2.00 \pm 0.50}$ & $2.05 \pm 0.20^{\mathrm{a}}$ \\
\hline$\rightarrow 9-11$ & $\underline{34}$ & $\underline{2.78 \pm 0.58}$ & $\underline{2.18 \pm 0.53}$ & $2.43 \pm 0.66$ & $\underline{1.86 \pm 0.66}$ & $2.32 \pm 0.29^{\mathrm{ab}}$ \\
\hline$\geq \geq 11$ & $\underline{47}$ & $\underline{3.14 \pm 0.66}$ & $\underline{3.27 \pm 0.45}$ & $2.33 \pm 0.45$ & $\underline{2.60 \pm 0.55}$ & $2.81 \pm 0.27^{\mathrm{b}}$ \\
\hline Overall & $\underline{319}$ & $2.57 \pm 0.22$ & $\underline{2.61 \pm 0.15}$ & $2.16 \pm 0.18$ & $2.72 \pm 0.20$ & $2.57 \pm 0.02$ \\
\hline
\end{tabular}

Means in the same column with different superscripts differ significantly $(\mathrm{P}<0.05)$.

Table 8: Influence of camel breeds and calving seasons on the calving interval/months (Mean $\pm \mathrm{SE}$ )

\begin{tabular}{|c|c|c|c|c|c|c|}
\hline \multirow{2}{*}{$\begin{array}{l}\frac{\text { Successive }}{\text { Calving }} \\
\underline{\text { Seasons }} \\
\end{array}$} & \multirow{2}{*}{$\frac{\text { No. of }}{\underline{\text { Animals }}}$} & \multicolumn{4}{|c|}{$\underline{\text { Camel Breeds }}$} & \multirow{2}{*}{$\frac{\text { overall mean }}{\underline{(\mathrm{n}=110)}}$} \\
\hline & & $\frac{\text { Magahiem }}{(\mathrm{n}=19)}$ & $\frac{\text { Maghatier }}{(\mathrm{n}=38)}$ & $\begin{array}{l}\underline{\text { Safrah }} \\
(\mathrm{n}=27) \\
\end{array}$ & $\begin{array}{l}\text { Hamrah } \\
\underline{(\mathrm{n}=26)}\end{array}$ & \\
\hline$\underline{2}^{\text {nd }}$ & $\underline{25}$ & $\underline{18.29 \pm 1.31}$ & $\underline{21.06 \pm 1.15}$ & $\underline{19.40 \pm 1.55}$ & $\underline{18.38 \pm 1.73}$ & $\underline{19.52 \pm 1.04}$ \\
\hline
\end{tabular}

21 


\begin{tabular}{|c|}
\hline $\begin{array}{l}\text { Formatted: Font: } 10 \mathrm{pt} \text {, Complex } \\
\text { Script Font: } 14 \mathrm{pt}\end{array}$ \\
\hline $\begin{array}{l}\text { Formatted: Justify Low, Left-to-right, } \\
\text { After: } 0 \mathrm{~cm}\end{array}$ \\
\hline Formatted: Left-to-right \\
\hline $\begin{array}{l}\text { Formatted: Justify Low, Left-to-right, } \\
\text { After: } 0 \mathrm{~cm}\end{array}$ \\
\hline $\begin{array}{l}\text { Formatted: Font: } 8 \text { pt, Bold, } \\
\text { Underline, Complex Script Font: } 8 \text { pt, } \\
\text { Not Bold, (Complex) Arabic (Egypt) }\end{array}$ \\
\hline $\begin{array}{l}\text { Formatted: Justify Low, Left-to-right, } \\
\text { After: } 0 \mathrm{~cm}\end{array}$ \\
\hline Formatted: Centered \\
\hline Formatted: Centered \\
\hline Formatted: Centered \\
\hline Formatted: Centered \\
\hline Formatted: Highlight \\
\hline Formatted: Centered \\
\hline Formatted: Not Highlight \\
\hline Formatted: Centered \\
\hline
\end{tabular}

Formatted: Font: $1 \mathrm{pt}$, Complex Script Font: 1 pt

Formatted: Left-to-right

Formatted: Font: 4 pt, Complex Script Font: 4 pt

Formatted: Justify Low, Indent: First line: $0 \mathrm{~cm}$, Line spacing: single

Formatted: Font: $10 \mathrm{pt}$, Complex Script Font: $10 \mathrm{pt}$

Formatted: Font: $11 \mathrm{pt}$, Complex Script Font: $11 \mathrm{pt}$

Formatted: Indent: First line: $0 \mathrm{~cm}$, Space Before: 0 pt, Line spacing:

single

Formatted: Footer distance from edge: $2 \mathrm{~cm}$, Number of columns: 2

Formatted: Font: $10 \mathrm{pt}$, Complex Script Font: $10 \mathrm{pt}$

Formatted: Space Before: $0 \mathrm{pt}$, Line spacing: single

Formatted: Justify Low, Indent: First line: $0 \mathrm{~cm}$, Line spacing: single

Formatted: Font: $10 \mathrm{pt}$, Complex

Script Font: $10 \mathrm{pt}$

Formatted: Font: $12 \mathrm{pt}$, Bold

Complex Script Font: 14 pt, Bold

Formatted: Left-to-right

Formatted: Font: $12 \mathrm{pt}$, Bold,

Complex Script Font: 14 pt, Bold

$\rightarrow$

2

$\rightarrow$ Assiut Vet. Med. J. Vol. 58 No. 135 October 2012

\begin{tabular}{lllllll}
\hline$\underline{3^{\text {rd }}}$ & $\underline{22}$ & $\underline{18.33 \pm 1.73}$ & $\underline{19.74 \pm 1.15}$ & $\underline{18.19 \pm 1.31}$ & $\underline{20.17 \pm 2.45}$ & $\underline{19.03 \pm 0.51}$ \\
\hline$\underline{4^{\text {th }}}$ & $\underline{29}$ & $\underline{18.50 \pm 3.46}$ & $\underline{18.80 \pm 1.10}$ & $\underline{19.03 \pm 3.14}$ & $\underline{19.50 \pm 1.10}$ & $\underline{19.09 \pm 0.46}$ \\
\hline$\underline{\underline{5}^{\text {th }}}$ & $\underline{12}$ & $\underline{22.80 \pm 1.73}$ & $\underline{21.87 \pm 2.00}$ & $\underline{20.40 \pm 2.45}$ & $\underline{16.73 \pm 2.00}$ & $\underline{20.55 \pm 1.03}$ \\
\hline$\underline{\underline{6}^{\text {th }}}$ & $\underline{13}$ & $\underline{17.89 \pm 2.00}$ & $\underline{22.83 \pm 1.41}$ & $\underline{19.92 \pm 2.45}$ & $\underline{25.67 \pm 2.45}$ & $\underline{21.68 \pm 0.95}$ \\
\hline$\underline{\underline{2}}$ & $\underline{9}$ & $\underline{\text { No Data }}$ & $\underline{20.86 \pm 2.00}$ & $\underline{20.28 \pm 2.00}$ & $\underline{19.09 \pm 1.55}$ & $\underline{19.69 \pm 0.70}$ \\
\hline$\underline{\text { Total }}$ & $\underline{110}$ & $\underline{19.19 \pm 1.00}$ & $\underline{20.49 \pm 0.56}$ & $\underline{19.19 \pm 0.67}$ & $\underline{19.46 \pm 0.56}$ & $\underline{19.70 \pm 0.34}$ \\
\hline
\end{tabular}

Fig.(1): Distribution of calving percentages

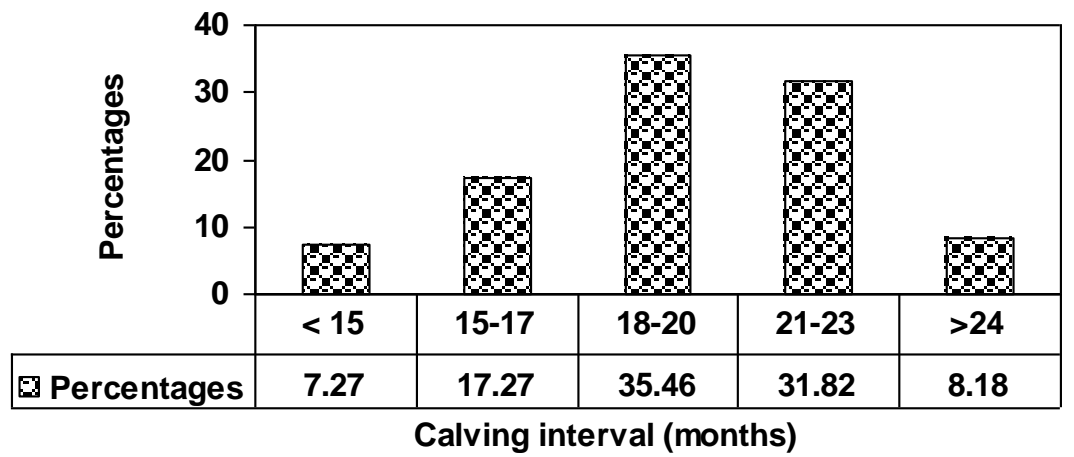

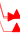

\section{DISCUSSION}

$\rightarrow$ The reproductive function beginning by onset of puberty is -affected remarkably by change in body weight. Attainment of puberty is influenced by the overall growth and weight of the animal which in turn is affected by nutrition (Marai et al. 2007). -Ages at first service, conception and calving may constitute major reasons for the long generation interval in camels. In the present study, the overall mean values of ages and weights at first mating, conception and calving were $39.75,41.82,54.39$ months; 437.17 , 450.16 and $519.03 \mathrm{~kg}$, respectively. These results were in agreement with those reported ir countries as Magrebi Arabia, where the a conception and first calving were reportec and 49.4 months, respectively (Sghir Moreover, Mounir and Borni (2012) recorr first successful mating of Maghreby $\mathrm{Ne}_{\text {i }}$ varied between 33 to 48 months with an ave months and the age at first parturition varie 68 months. In Turkmenistan, Arvana can for the first time at 3 years of age and 350 . live weight (Dmitriez and Ernst, 1989). In $66.7 \%$ of the female camels were bred at age (Abbas and Musa, 1989). In the Ur 


\begin{tabular}{|c|}
\hline $\begin{array}{l}\text { Formatted: Font: } 10 \mathrm{pt} \text {, Complex } \\
\text { Script Font: } 14 \mathrm{pt}\end{array}$ \\
\hline $\begin{array}{l}\text { Formatted: Justify Low, Left-to-right, } \\
\text { After: } 0 \mathrm{~cm}\end{array}$ \\
\hline Formatted: Left-to-right \\
\hline $\begin{array}{l}\text { Formatted: Justify Low, Left-to-right, } \\
\text { After: } 0 \mathrm{~cm}\end{array}$ \\
\hline $\begin{array}{l}\text { Formatted: Font: } 8 \text { pt, Bold, } \\
\text { Underline, Complex Script Font: } 8 \text { pt, } \\
\text { Not Bold, (Complex) Arabic (Egypt) }\end{array}$ \\
\hline $\begin{array}{l}\text { Formatted: Justify Low, Left-to-right, } \\
\text { After: } 0 \mathrm{~cm}\end{array}$ \\
\hline ... matted \\
\hline ... matted \\
\hline ... matted \\
\hline ... matted \\
\hline ... matted \\
\hline ... matted \\
\hline ... matted \\
\hline ... matted \\
\hline ... matted \\
\hline ... matted \\
\hline ... matted \\
\hline ... matted \\
\hline ... matted \\
\hline ... matted \\
\hline ... matted \\
\hline ... matted \\
\hline ... matted \\
\hline ... matted \\
\hline ... matted \\
\hline ... matted \\
\hline ... matted \\
\hline ... matted \\
\hline ... matted \\
\hline ... matted \\
\hline ... matted \\
\hline ... matted \\
\hline ... matted \\
\hline ... matted \\
\hline ... matted \\
\hline ... matted \\
\hline ... matted \\
\hline ... matted \\
\hline Formatted: Left-to-right \\
\hline ... matted \\
\hline
\end{tabular}

$\rightarrow$ Assiut Vet. Med. J. Vol. 58 No. 135 October 2012

Emirates, ages at first service and calving were $43.1 \pm 0.58$ and $55.3 \pm 2.87$ months, respectively $\rightarrow$ (Aboul-Ela 1991). In Libya, ages at first service and first calving were $36.9 \pm 1.10$ and $50.3 \pm 1.28$ months, respectively (Hermas and Shareha, 1991). In contrast, the current findings were less than 61-62 months which were reported for Bikaneri camels as age at first calving (Beniwal and Chaudhry, 1984 and Khanna et al. 1990). Moreover, in Pakistan (Yasin and Wahid, 1957); Indian (Khanna et al. 1990); Sudan (Köhler Kohler-Rollefson et al. 1990); Horn of Africa (Hartley, (1984) and Kenya (Karimi and Kimenye, 1990) the she-camels were reported to reach puberty at 4-5 years of age. The variation in these observations may be related to feeding and management and/or breed differences. However, the onset of puberty is remarkably independent on body weight. Therefore, dromedary camels reproductive performance in terms of age at puberty, at first conception and at first birth can be improved by ensuring adequate nutrition in early life and improvement of managerial and environmental conditions as well as by using hormonal treatment that can assist early sexual development and breeding maturity (Arthur and Al-Rahim, 1982; Simpkin, 1987; Zaied, 1991; Formigoni et al. 1996; and Mounir and Borni, 2012).

The overall mean of the interval between services within the same breeding season which starts at early November to late April was $19.32 \pm 0.26$ days. The present findings agree with those recorded by ELAzab et al. (1997) where the mean interval between services within the same breeding season was $15.83 \pm 0.39$ days.

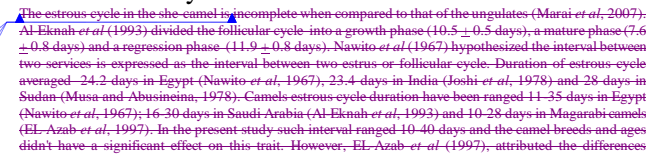

The overall period from ealving to first onset hea (postpartum period) was $45.39 \pm 2.57$ days. Similarly, other studies indicating that the postpartum heat occurs 14-30 days after calving (Novoa, 1970; Evans and Powys, 1979; Abdel-Rahim and El-Nazier, 1992) and between 10-72 days with mean 26.34 \pm 1.89 days for Magarabi female camels (EL-Azab et al. 1997). In this study, it has been observed that the majority of female camels calved at the beginning of a breeding season came into the first postpartum heat after 13 103 days after calving within the same breeding season. It is importance to notice that in camels even with the early induction of heat after calving, the majority of females fail to conceive within the same breeding season, and this might be due to the effects of lactation, feeding status of the animals, body weight and conformation and feed availability
(Shalash 1965; Shareha et al.. 1982; Artl and Mounir and Borni, 2012).

However, Hermas et al (1990) noticed the 1 heat in Magarabi camels to oceur 233.97士 after calving and authors attributed this lon to the delay in estrus detection for the females till the fortheoming breeding seaser The overall mean of service periods -was 7 days, came relative to value $51.9 \pm 12.1 \mathrm{~d}$ recorded by Hermas and Shareha (1991) i camel. In the present study, The overall me: days herein was $317.61 \pm 4.54$ days, in accor EL-Azab et al. (1997) and Hermas et whereas open days period elapsed from conception for Magarabi female car $308.02 \pm 6.95$ days and $286.80 \pm 12.70$ days. $\mathrm{F}$ recent study recorded shorter interval (147표 between calving to successful mating of Negga camel (Mounir and Borni, 2012)..

The overall mean value of the nı services/conception was $2.57 \pm 0.02$ Similarly, in Libya, Hermas and Share] reported that services/conception was while in United Arab Emirates, Aboul-E showed that the services/conception were and the conception percentage occurred service was $58 \%$ and only $20 \%$ of she came $\geq 3$ services before pregnancy. In this study the available observations it has been obser Saudi Arabia, camels' breeding season is st early November to late April and it may early May. During this period both males a1 are fertile. On the other hand, summer mont September) are considered as a non-breed for local camel breeds (male and female reproductive activities of camels were adversely by the heat stress and the funct high ambient temperature -(Habeeb et c Marai and Habeeb, 1998 and Marai et al, 2 Generally, in this study, the traits of serv (days) and number of services/concep significantly affected by months of mati been observed that, service period and 1 services/conception were significantly less during November to January $(43.74 \pm 2.39$ $2.22 \pm 0.12$ services) than for that during $F$ April (77.68 \pm 3.27 days and $3.75 \pm 0.16$ (untabulated data). Thus, the pattern of $t$ reproductive cycle appears to relatec environment in which they live (Novoa, 1 breeding season differs in the various co Pakistan (Yasin and Wahid, 1957), China (1 1985), Egypt (Shalash, 1965) and Israel ( Etzion, 1980), the breeding season of camel from December to April. In Somalia, Ma 


\begin{tabular}{|c|}
\hline $\begin{array}{l}\text { Formatted: Font: } 10 \mathrm{pt} \text {, Complex } \\
\text { Script Font: } 14 \mathrm{pt}\end{array}$ \\
\hline $\begin{array}{l}\text { Formatted: Justify Low, Left-to-right, } \\
\text { After: } 0 \mathrm{~cm}\end{array}$ \\
\hline Formatted: Left-to-right \\
\hline $\begin{array}{l}\text { Formatted: Justify Low, Left-to-right, } \\
\text { After: } 0 \mathrm{~cm}\end{array}$ \\
\hline $\begin{array}{l}\text { Formatted: Font: } 8 \text { pt, Bold, } \\
\text { Underline, Complex Script Font: } 8 \text { pt, } \\
\text { Not Bold, (Complex) Arabic (Egypt) }\end{array}$ \\
\hline $\begin{array}{l}\text { Formatted: Justify Low, Left-to-right, } \\
\text { After: } 0 \mathrm{~cm}\end{array}$ \\
\hline $\begin{array}{l}\text { Formatted: Font: } 10 \mathrm{pt} \text {, Complex } \\
\text { Script Font: } 10 \mathrm{pt}\end{array}$ \\
\hline
\end{tabular}

\begin{tabular}{|c|}
\hline ... matted \\
\hline $\begin{array}{l}\text { Formatted: Font: } 10 \mathrm{pt} \text {, Complex } \\
\text { Script Font: } 10 \mathrm{pt}\end{array}$ \\
\hline $\begin{array}{l}\text { Formatted: Font: } 11 \mathrm{pt} \text {, Complex } \\
\text { Script Font: } 11 \mathrm{pt}\end{array}$ \\
\hline $\begin{array}{l}\text { Formatted: Centered, Line spacing: } \\
\text { single }\end{array}$ \\
\hline $\begin{array}{l}\text { Formatted: Font: } 10 \mathrm{pt} \text {, Complex } \\
\text { Script Font: } 14 \mathrm{pt}\end{array}$ \\
\hline $\begin{array}{l}\text { Formatted: Normal, Line spacing: } \\
\text { single }\end{array}$ \\
\hline $\begin{array}{l}\text { Formatted: Justify Low, Indent: First } \\
\text { line: } 0 \mathrm{~cm} \text {, Line spacing: single }\end{array}$ \\
\hline $\begin{array}{l}\text { Formatted: Justify Low, Line spacing: } \\
\text { single }\end{array}$ \\
\hline $\begin{array}{l}\text { Formatted: Font: } 11 \mathrm{pt} \text {, Complex } \\
\text { Script Font: } 11 \mathrm{pt}\end{array}$ \\
\hline Formatted: Line spacing: single \\
\hline $\begin{array}{l}\text { Formatted: Font: } 10 \mathrm{pt} \text {, Complex } \\
\text { Script Font: } 14 \mathrm{pt}\end{array}$ \\
\hline $\begin{array}{l}\text { Formatted: Font: } 10 \mathrm{pt} \text {, Italic, } \\
\text { Complex Script Font: } 10 \mathrm{pt} \text {, Italic }\end{array}$ \\
\hline $\begin{array}{l}\text { Formatted: Justify Low, Indent: } \\
\text { Hanging: } 2.7 \mathrm{ch} \text {, Left } 0 \mathrm{ch} \text {, First line: } \\
-2.7 \mathrm{ch} \text {, Line spacing: single }\end{array}$ \\
\hline ... matted \\
\hline ... matted \\
\hline $\begin{array}{l}\text { Formatted: Font: } 5 \mathrm{pt} \text {, Complex Script } \\
\text { Font: } 5 \mathrm{pt}\end{array}$ \\
\hline $\begin{array}{l}\text { Formatted: Font: } 12 \mathrm{pt} \text {, Bold, } \\
\text { Complex Script Font: } 14 \text { pt, Bold }\end{array}$ \\
\hline Formatted: Left-to-right \\
\hline $\begin{array}{l}\text { Formatted: Font: } 12 \mathrm{pt} \text {, Bold, } \\
\text { Complex Script Font: } 14 \mathrm{pt} \text {, Bold }\end{array}$ \\
\hline
\end{tabular}

\section{$\rightarrow$ Assiut Vet. Med. J. Vol. 58 No. 135 October 2012}

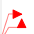

observed that the male camel ruts in the spring months (April and May). In India, the breeding period is from November to February (Singh and Prakash, 1964). Contrariwise, in the Sudan, Musa and Abusinea (1978) reported the breeding season to be from March to August. Similarly, in Eritrea the breeding of camels starts at the beginning of the rainy season in July and continues throughout, but if camels are in god condition and the plenty of forage is available, the breeding males become sexually active and females are fertile and receptive at any season (Gebrehiwet, 1997). It was also noted that the majority of the females in the herd were mated in the summer (rainy seasen, commeneing in July) and the rest in the winter (short rainy season, commencing in January) when forage was plentiful (Marai and Habeeb, 1998). Long calving intervals are the most major factor contributing to poor reproductive performance of camels. Under extensive management system calving interval lasts for more than 24 months (Evans and Powys, 1979). However, the calving interval of camels may reach eighteen months, similar to that of cows (Knoess, 1976). The overall mean of calving interval was $19.70+0.34$ months; and no significant difference in the calving interval between camel breeds and successive calving seasons. These results are in agreement with that reported by Basmaeil et al. (1994) in Saudi Arabia where the calving interval for five successive breeding seasons was $20.58 \pm 0.82$ months. Similarly, Mounir and Borni, (2012) cited that, the intervals between calving of Maghreby Negga camel was $526 \pm 145$ days. Moreover, Dmitriez and Ernst (1989) in Turkmenistan $\rightarrow$ obtained in theirrir study- 2 calves/3years. As well as, -in Kenya, Evans and Powys (1979) observed -an -average calving interval of 22 months if young survives. In the same purport, the present findings were longer than those reported by Richard et al. (1985) in Niger (15.0 months); Mosleh (1991) in Tunisia (13.45+0.27 months) and Köhler-Rollefson (1991) in Sudan (14-15 months). In contrast, the present calving interval was shorter than those recorded by Aboul-Ela (1991) in United Arab Emirates (24.4+0.68 months); Khanna et al. (1990) in Indian (25.73 \pm 0.27 months); Hermas and Shareha (1991) in Magrebi Arabia countries $(22.62 \pm 0.40$ to 24.0+8.2 months); Dioli (1991) in East Africa (24.0 months); Aslam et al. (2002) in Pakistan (23.5 1.33 months); Schwartz et al. (1983) in Kenya (28 months) and Herren (1993) in Somalia -(29 months). The disagreement of these observations was attributed to differences in she camels' gestation length and seasonality of breeding (Wilson, 1984 and Arthur et al $_{2}$ 1985); late post-partum estrus (Mukasa-Mugerwa, 1981) and individual variation in open days period (Aboul-Ela, 1991)

$$
\longrightarrow
$$

2

Among the studied camel herd, the calvir distribution classes were $<15,15-17,18-20$, $>24$ months with corresponding percent: $7.27 \%, 17.27 \%, 35.46 \%, 31.82 \%$ and 8.1 she-camels, respectively. Similarly, in M (1979) reported that, $20.9 \%, 27.9 \%, 44.2 \%$ of a herd of she-camels showed calving is ranges 12-15, 16-23, 24-25 and >25 respectively. Also, in United Arab Emiratı Ela (1991) indicated that the intervals calving is $<20$ months for $14.4 \%$ of $\mathrm{s}$. Moreover, in Kenya, Bremaud (1969) den $11.5 \%, 3.9 \%, 53.5 \%$ and $30.8 \%$ of she-ci with calving intervals $12-15,16-23,24-2$ s months, respectively.. Herren (1993) repor Somalia -a period of 28 months was estir calving interval in $35-40 \%$ of a herd of Generally, the current calving interval show of calving intervals. Short calving interv average 14 months that was observed to cor breeding female camels aborted at late pregnancy and in cases of calf death after $d$ this case, the dam was submitted to a bull conception within one month. The mediu interval (between 16 and 18 months) was the female camels delivered at the beginn breeding season, and calves were weaned : 75 days of age, and their dams were $r$ became pregnant at the end of the same sec calving interval ( $\geq 23$ months) was observec female camels were kept milking to satisf demand of the calves.

\section{CONCLUSION}

Reproductive traits, in terms of post-partı service period and open days are depend camel breeds, and this indicates the imp heritability as a value that express anc average additive gene effect. Howeve research is -needed to determine the between genetic merits of camel breeds productivity including reproductive pes which may be better in some camel br others.

\section{REFERENCES}

Abbas, B. and Musa, B.E. (1989)-: Obser camel husbandry with special em reproductive performance of the fen in Northern Butana-Sudan. Camel I (5): 8. $\frac{\text { Abdallah, H.R. and Bernard Faye. }}{\text { Phenotypic classification of }}$ Arabiancamel (Camelus dromedariu: body measurements. Emir. J. Food (3): 272-280. 


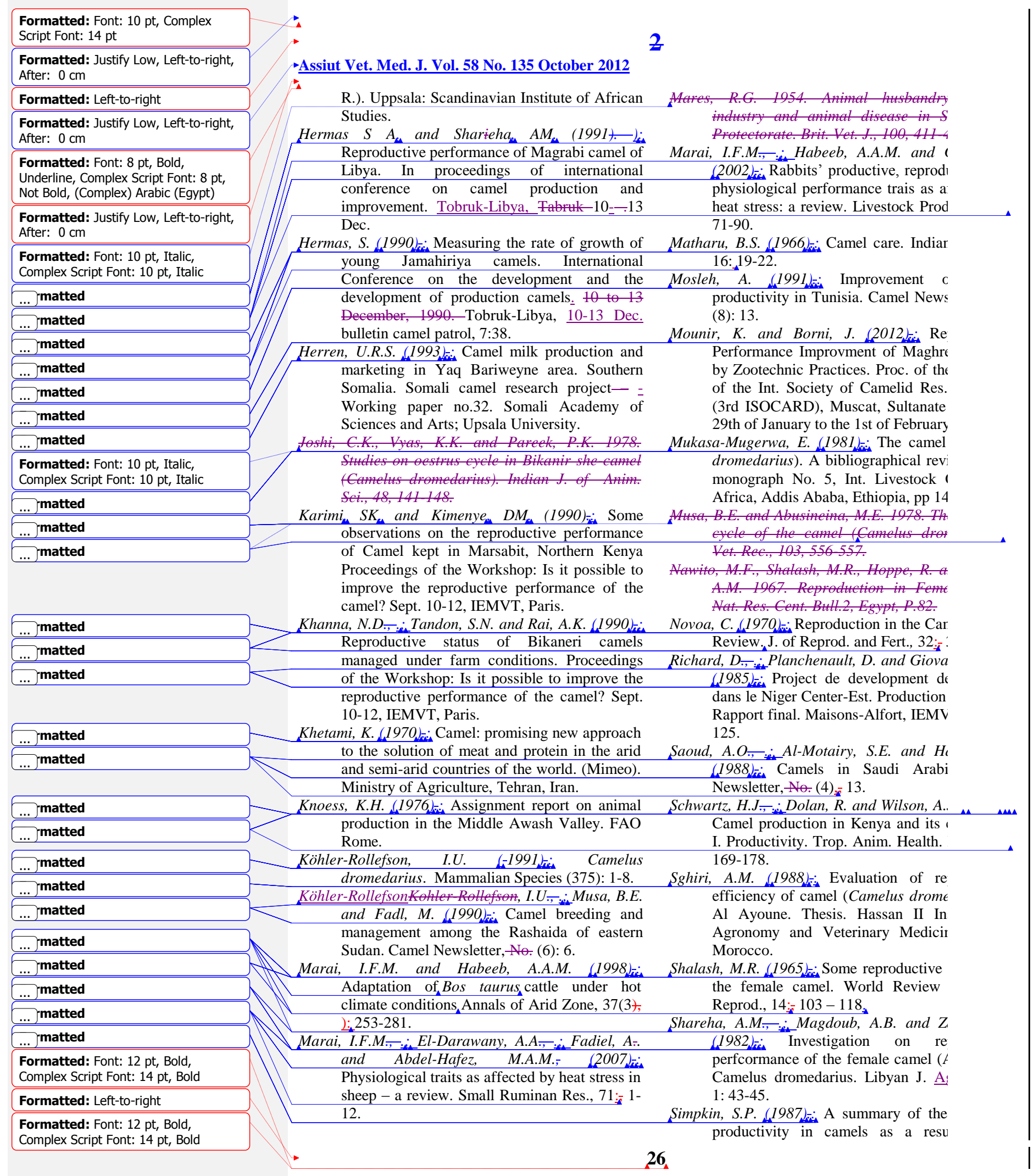




\begin{tabular}{|c|}
\hline $\begin{array}{l}\text { Formatted: Font: } 10 \mathrm{pt} \text {, Complex } \\
\text { Script Font: } 14 \mathrm{pt}\end{array}$ \\
\hline $\begin{array}{l}\text { Formatted: Justify Low, Left-to-right, } \\
\text { After: } 0 \mathrm{~cm}\end{array}$ \\
\hline Formatted: Left-to-right \\
\hline $\begin{array}{l}\text { Formatted: Justify Low, Left-to-right, } \\
\text { After: } 0 \mathrm{~cm}\end{array}$ \\
\hline $\begin{array}{l}\text { Formatted: Font: } 8 \text { pt, Bold, } \\
\text { Underline, Complex Script Font: } 8 \text { pt, } \\
\text { Not Bold, (Complex) Arabic (Egypt) }\end{array}$ \\
\hline $\begin{array}{l}\text { Formatted: Justify Low, Left-to-right, } \\
\text { After: } 0 \mathrm{~cm}\end{array}$ \\
\hline Formatted: Justify Low \\
\hline Formatted: Justify Low \\
\hline Formatted: Justify Low \\
\hline Formatted: Justify Low \\
\hline Formatted: Justify Low \\
\hline Formatted: Line spacing: single \\
\hline ... matted \\
\hline Formatted: Justify Low \\
\hline Formatted Table \\
\hline Formatted: Justify Low \\
\hline Formatted: Justify Low \\
\hline Formatted: Justify Low \\
\hline Formatted: Justify Low \\
\hline Formatted: Justify Low \\
\hline Formatted: Justify Low \\
\hline Formatted: Justify Low \\
\hline Formatted: Line spacing: single \\
\hline Formatted: Justify Low \\
\hline Formatted Table \\
\hline Formatted: Justify Low \\
\hline Formatted: Justify Low \\
\hline Formatted: Justify Low \\
\hline Formatted: Justify Low \\
\hline Formatted: Justify Low \\
\hline Formatted: Justify Low \\
\hline
\end{tabular}

$\rightarrow$ Assiut Vet. Med. J. Vol. 58 No. 135 October 2012

\begin{tabular}{lllllll|}
\hline$\rightarrow 7-7$ & 105 & $17.93 \pm 1.65$ & $19.22 \pm 0.89$ & $19.58 \pm 1.84$ & $17.65 \pm 1.55$ & $18.78 \pm 0.63$ \\
\hline$\rightarrow 9-11$ & 72 & $21.86 \pm 1.20$ & $18.88 \pm 1.11$ & $18.00 \pm 4.51$ & $21.29 \pm 1.55$ & $20.41 \pm 0.84$ \\
\hline$\rightarrow 11$ & 81 & $20.09 \pm 1.13$ & $18.92 \pm 1.84$ & $21.00 \pm 2.02$ & $16.62 \pm 1.77$ & $19.34 \pm 0.84$ \\
\hline 9verall & 687 & $20.61 \pm 1.20$ & $18.17 \pm 1.50$ & $18.20 \pm 2.85$ & $17.45 \pm 1.43$ & $18.93 \pm 0.90$ \\
\hline
\end{tabular}

$\rightarrow$ Table 4: Influence of camel breeds and ages on the post partmm period (MeantSE)

\begin{tabular}{|c|c|c|c|c|c|c|}
\hline \multirow{2}{*}{$\rightarrow$ Ages } & \multirow{2}{*}{$\begin{array}{l}\text { No. of } \\
\text { ebservations }\end{array}$} & \multicolumn{4}{|c|}{$\begin{array}{l}\text { Camel Breeds } \\
\text { (Number of observations) }\end{array}$} & \multirow{2}{*}{$\begin{array}{l}\text { Overall } \\
(\text { no. }=108)\end{array}$} \\
\hline & & $\begin{array}{l}\text { Magahiem } \\
(\text { no. }=25)\end{array}$ & $\begin{array}{l}\text { Maghatier } \\
(\text { no. }=32)\end{array}$ & $\begin{array}{l}\text { Safrah } \\
\text { (no.=26) }\end{array}$ & $\begin{array}{l}\text { Hamrah } \\
\text { (no.=25) }\end{array}$ & \\
\hline$\leq 5$ & 23 & $34.67 \pm 12.14$ & $38.67 \pm 8.57$ & $42.00 \pm 12.11$ & $29.00 \pm 12.14$ & $36.29 \pm 5.05$ \\
\hline$\rightarrow 5-7$ & 20 & $70.00 \pm 12.11$ & $51.00 \pm 12.12$ & $51.00 \pm 12.11$ & $33.33 \pm 12.00$ & $51.33 \pm 6.34$ \\
\hline$\rightarrow 7-9$ & 22 & $45.00 \pm 12.11$ & $40.67 \pm 12.00$ & $32.00 \pm 14.84$ & $35.40 \pm 9.39$ & $38.31 \pm 4.54$ \\
\hline$\rightarrow 9-11$ & 21 & $58.00 \pm 12.11$ & $58.00 \pm 12.17$ & $69.00 \pm 12.11$ & $31.00 \pm 12.00$ & $54.00 \pm 6.61$ \\
\hline$\leftrightarrow \geq 11$ & 22 & $51.67 \pm 12.00$ & $40.00 \pm 12.12$ & $58.00 \pm 9.39$ & $48.50 \pm 14.84$ & $50.92 \pm 5.21$ \\
\hline$\rightarrow \theta$ verall & 108 & $48.67 \pm 4.51^{\mathrm{a}}$ & $46.64 \pm 5.44^{\text {ab }}$ & $52.74 \pm 4.38^{a}$ & $36.83 \pm 3.73^{b}$ & $45.39 \pm 2.57$ \\
\hline
\end{tabular}

$\rightarrow \quad$ Means in the same row with different superseripts differ significantly $(\mathrm{P}<0.05)$.

$\rightarrow$

Table 5: Influence of camel breeds and ages on the service periods/days (Mean \pm SE) Eamel Breeds

\begin{tabular}{|c|c|c|c|c|c|c|}
\hline \multirow{2}{*}{$\rightarrow$ Ages } & \multirow{2}{*}{$\begin{array}{l}\text { No. of } \\
\text { ebservations }\end{array}$} & \multicolumn{4}{|c|}{ (Number of observations) } & \multirow{2}{*}{$\begin{array}{l}\text { Overall } \\
\text { (no.=197) }\end{array}$} \\
\hline & & $\begin{array}{l}\text { Magahiem } \\
(\text { no. }=39)\end{array}$ & $\begin{array}{l}\text { Maghatier } \\
\text { (no.=44) }\end{array}$ & $\begin{array}{l}\text { Safrah } \\
(\mathrm{no} 0 .=56)\end{array}$ & $\begin{array}{l}\text { Hamrah } \\
\text { (no. }=58)\end{array}$ & \\
\hline$\rightarrow \leq 5$ & 52 & $45.00 \pm 15.35$ & $48.14 \pm 12.97$ & $70.00 \pm 15.35$ & $87.44 \pm 11.44$ & $65.35 \pm 6.38^{b}$ \\
\hline$\rightarrow 5-7$ & 50 & $75.00 \pm 17.16$ & $44.00 \pm 15.35$ & $82.88 \pm 12.14$ & $100.88 \pm 12.14$ & $79.60 \pm 8.05^{\text {ab }}$ \\
\hline$\rightarrow 7-9$ & 40 & $73.50 \pm 17.20$ & $86.00 \pm 17.18$ & $51.75 \pm 17.16$ & $95.25 \pm 12.10$ & $80.35 \pm 8.17^{\text {ab }}$ \\
\hline$\rightarrow-11$ & 30 & $82.75 \pm 17.16$ & $56.67 \pm 19.82$ & $43.00 \pm 15.35$ & $66.00 \pm 19.82$ & $60.93 \pm 7.20^{-b}$ \\
\hline$\rightarrow \geq 11$ & 25 & $82.40 \pm 21.71$ & $113.67 \pm 19.82$ & $73.33 \pm 14.01$ & $149.00 \pm 34.33$ & $90.88 \pm 10.85^{*}$ \\
\hline werall & 197 & $69.54 \pm 7.41^{b}$ & $64.18 \pm 7.62^{b}$ & $66.96 \pm 5.80^{b}$ & $93.21 \pm 7.42^{\mathrm{a}}$ & $74.58 \pm 3.62$ \\
\hline
\end{tabular}

Means in the same column with different superseripts differ significantly ( $(\mathrm{P}<0.05)$

Means in the same row with different superscripts differ significantly $(P<0.05)$.

Formatted: Line spacing: single

Table 6: Influence of camel breeds and ages on the open days period (Mean \pm SE)

Formatted: Justify Low

Formatted Table

Formatted: Justify Low

Formatted: Justify Low

Formatted: Justify Low

matted

Formatted: Left-to-right

matted

\begin{tabular}{|c|c|c|c|c|c|c|}
\hline \multirow{2}{*}{ Arges } & \multirow{2}{*}{$\begin{array}{l}\text { No. of } \\
\text { ebservations }\end{array}$} & \multicolumn{4}{|c|}{$\begin{array}{l}\text { Camel Breeds } \\
\text { (Number of observations) }\end{array}$} & \multirow{2}{*}{$\begin{array}{l}\text { Overall } \\
(\text { no. }=272)\end{array}$} \\
\hline & & $\begin{array}{l}\text { Magahiem } \\
\text { (no.=46) }\end{array}$ & $\begin{array}{l}\text { Maghatier } \\
(\mathrm{no} .=81)\end{array}$ & $\begin{array}{l}\text { Safrah } \\
(\text { no.=63) }\end{array}$ & $\begin{array}{l}\text { Hamrah } \\
(\mathrm{no}=82)\end{array}$ & \\
\hline$\leq 5$ & 78 & $300.71 \pm 17.32$ & $345.88 \pm 12.96$ & $343.54 \pm 13.23$ & $282.60 \pm 16.73$ & $324.89 \pm 7.28^{\text {be }}$ \\
\hline $5=7$ & 55 & $365.40 \pm 28.97$ & $356.40 \pm 14.49$ & $336.29 \pm 17.32$ & $323.25 \pm 16.20$ & $342.46 \pm 7.59^{\mathrm{e}}$ \\
\hline $7 \rightarrow 9$ & 45 & $305.40 \pm 28.97$ & $262.75 \pm 22.91$ & $275.80 \pm 28.97$ & $327.00 \pm 22.91$ & $293.23 \pm 17.59^{\text {at }}$ \\
\hline
\end{tabular}


Formatted: Font: $10 \mathrm{pt}$, Complex

Script Font: 14 pt

Formatted: Justify Low, Left-to-right, After: $0 \mathrm{~cm}$

Formatted: Left-to-right

Formatted: Justify Low, Left-to-right, After: $0 \mathrm{~cm}$

Formatted: Font: 8 pt, Bold,

Underline, Complex Script Font: 8 pt,

Not Bold, (Complex) Arabic (Egypt)

Formatted: Justify Low, Left-to-right, After: $0 \mathrm{~cm}$

Formatted: Justify Low

Formatted: Justify Low

Formatted: Justify Low

Formatted: Justify Low

Formatted: Space Before: 0 pt, Line

spacing: single

Formatted: Justify Low

Formatted Table

Formatted: Justify Low

Formatted: Justify Low

Formatted: Justify Low

Formatted: Justify Low

Formatted: Justify Low

Formatted: Justify Low

Formatted: Justify Low, Line spacing:

single

... matted

Formatted: Justify Low

Formatted Table

Formatted: Justify Low

Formatted: Justify Low

Formatted: Justify Low

Formatted: Justify Low

Formatted: Justify Low

Formatted: Justify Low

\begin{tabular}{|l|l|}
\hline Formatted: Justify Low \\
\hline$\ldots$ & matted \\
\hline$\ldots$ & matted \\
\hline$\ldots$ & matted \\
\hline$\ldots$ & matted \\
\hline$\ldots$ & matted \\
\hline$\ldots$ & matted \\
\hline$\ldots$ & matted \\
\hline$\ldots$ & matto \\
\hline
\end{tabular}

... matted

Formatted: Left-to-right

matted $\longrightarrow$

\section{2}

$\rightarrow$ Assiut Vet. Med. J. Vol. 58 No. 135 October 2012

\begin{tabular}{|c|c|c|c|c|c|c|}
\hline $9 \cdot 11$ & 44 & $297.50 \pm 32.39$ & $301.40 \pm 28.97$ & $315.86 \pm 24.49$ & $227.67 \pm 21.60$ & $278.28 \pm 16.62^{-2}$ \\
\hline$¥ 11$ & 50 & $282.80 \pm 28.97$ & $317.65 \pm 13.51$ & $299.92 \pm 17.97$ & $327.22 \pm 21.59$ & $311.28 \pm 8.43^{-a b}$ \\
\hline Overall & 272 & $308.12 \pm 12.20^{\mathrm{ab}}$ & $329.51 \pm 7.50^{b}$ & $324.48 \pm 7.30^{\mathrm{ab}}$ & $297.82 \pm 8.63^{a t}$ & $317.61 \pm 4.54$ \\
\hline$\rightarrow$ Table & \multicolumn{6}{|c|}{$\begin{array}{l}\text { Means in the same column with different superseripts differ significantly }(\mathrm{P}<0.05) \text {. } \\
\text { Means in the same row with different superscripts differ significantly }(\mathrm{P}<0.05) \text {. } \\
\text { Influence of camel breeds and ages on the number of services/conception }(M e a n \pm S E)\end{array}$} \\
\hline \multirow{2}{*}{ Arges } & \multirow{2}{*}{$\begin{array}{l}\text { No. of } \\
\text { ebservations }\end{array}$} & \multicolumn{4}{|c|}{$\begin{array}{l}\text { Camel Breeds } \\
\text { (no. of observations) }\end{array}$} & \multirow{2}{*}{$\begin{array}{l}\text { Overall } \\
(\text { no. }=319)\end{array}$} \\
\hline & & $\begin{array}{l}\text { Magahiem } \\
(\mathrm{n \theta}=62)\end{array}$ & $\begin{array}{l}\text { Maghatier } \\
\text { (nө.=87) }\end{array}$ & $\begin{array}{l}\text { Safrah } \\
(\mathrm{ne}=82)\end{array}$ & $\begin{array}{l}\text { Hamrah } \\
\text { (ne:=88) }\end{array}$ & \\
\hline$\$ 5$ & 121 & $2.92 \pm 0.48$ & $2.76 \pm 0.26$ & $2.37 \pm 0.33$ & $3.39 \pm 0.29$ & $2.88 \pm 0.17^{b}$ \\
\hline $5-7$ & 74 & $2.53 \pm 0.45$ & $2.42 \pm 0.35$ & $2.19 \pm 0.43$ & $2.21 \pm 0.4 \theta$ & $2.34 \pm 0.18^{-a b}$ \\
\hline $7 \rightarrow 9$ & 43 & $2.44 \pm 0.58$ & $2.36 \pm 0.46$ & $1.13 \pm 0.61$ & $2.00 \pm 0.50$ & $2.05 \pm 0.2 \theta^{-2}$ \\
\hline $9=11$ & 34 & $2.78 \pm 0.58$ & $2.18 \pm 0.53$ & $2.43 \pm 0.66$ & $1.86 \pm 0.66$ & $2.32 \pm 0.29^{-a b}$ \\
\hline$¥ 11$ & 47 & $3.14 \pm 0.66$ & $3.27 \pm 0.45$ & $2.33 \pm 0.45$ & $2.60 \pm 0.55$ & $2.81 \pm 0.27^{b}$ \\
\hline Overall & 319 & $2.57 \pm 0.22$ & $2.61 \pm 0.15$ & $2.16 \pm 0.18$ & $2.72 \pm 0.20$ & $2.57 \pm 0.02$ \\
\hline
\end{tabular}

$\rightarrow \quad$ Means in the same column with different superscripts differ significantly ( $\mathrm{P}<0.05)$.

Fable 8: Influence of camel breeds and calving seasons on the calving interval/months (Mean \pm SE)

\begin{tabular}{|c|c|c|c|c|c|c|}
\hline \multirow{2}{*}{$\begin{array}{l}\text { Suceessive } \\
\text { Ealving } \\
\text { Seasens }\end{array}$} & \multirow{2}{*}{$\begin{array}{l}\text { No. of } \\
\text { Animals }\end{array}$} & \multicolumn{4}{|c|}{ Camel Breeds } & \multirow{2}{*}{$\begin{array}{l}\text { overall mean } \\
(\text { no. }=110)\end{array}$} \\
\hline & & $\begin{array}{l}\text { Magahiem } \\
(\text { no.=19) }\end{array}$ & $\begin{array}{l}\text { Maghatier } \\
(\text { no.=38) }\end{array}$ & $\begin{array}{l}\text { Safrah } \\
(\text { no.=27) }\end{array}$ & $\begin{array}{l}\text { Hamrah } \\
(\mathrm{no} .=26)\end{array}$ & \\
\hline $2^{\text {nd }}$ & 25 & $18.29 \pm 1.31$ & $21.06 \pm 1.15$ & $19.40 \pm 1.55$ & $18.38 \pm 1.73$ & $19.52 \pm 1.04$ \\
\hline $3^{\text {rd }}$ & 22 & $18.33 \pm 1.73$ & $19.74 \pm 1.15$ & $18.19 \pm 1.31$ & $20.17 \pm 2.45$ & $19.03 \pm 0.54$ \\
\hline $4^{\text {th }}$ & 29 & $18.50 \pm 3.46$ & $18.80 \pm 1.10$ & $19.03 \pm 3.14$ & $19.50 \pm 1.10$ & $19.09 \pm 0.46$ \\
\hline $5^{\text {th }}$ & 12 & $22.80 \pm 1.73$ & $21.87 \pm 2.0 \theta$ & $20.40 \pm 2.45$ & $16.73 \pm 2.09$ & $20.55 \pm 1.03$ \\
\hline $6^{\text {th }}$ & 13 & $17.89 \pm 2.00$ & $22.83 \pm 1.41$ & $19.92 \pm 2.45$ & $25.67 \pm 2.45$ & $21.68 \pm 0.95$ \\
\hline$\geq 7^{\text {th }}$ & 9 & No Data & $20.86 \pm 2.00$ & $20.28 \pm 2.00$ & $19.09 \pm 1.55$ & $19.69 \pm 0.70$ \\
\hline Total & 110 & $19.19 \pm 1.00$ & $20.49 \pm 0.56$ & $19.19 \pm 0.67$ & $19.46 \pm 0.56$ & $19.70 \pm 0.34$ \\
\hline
\end{tabular}

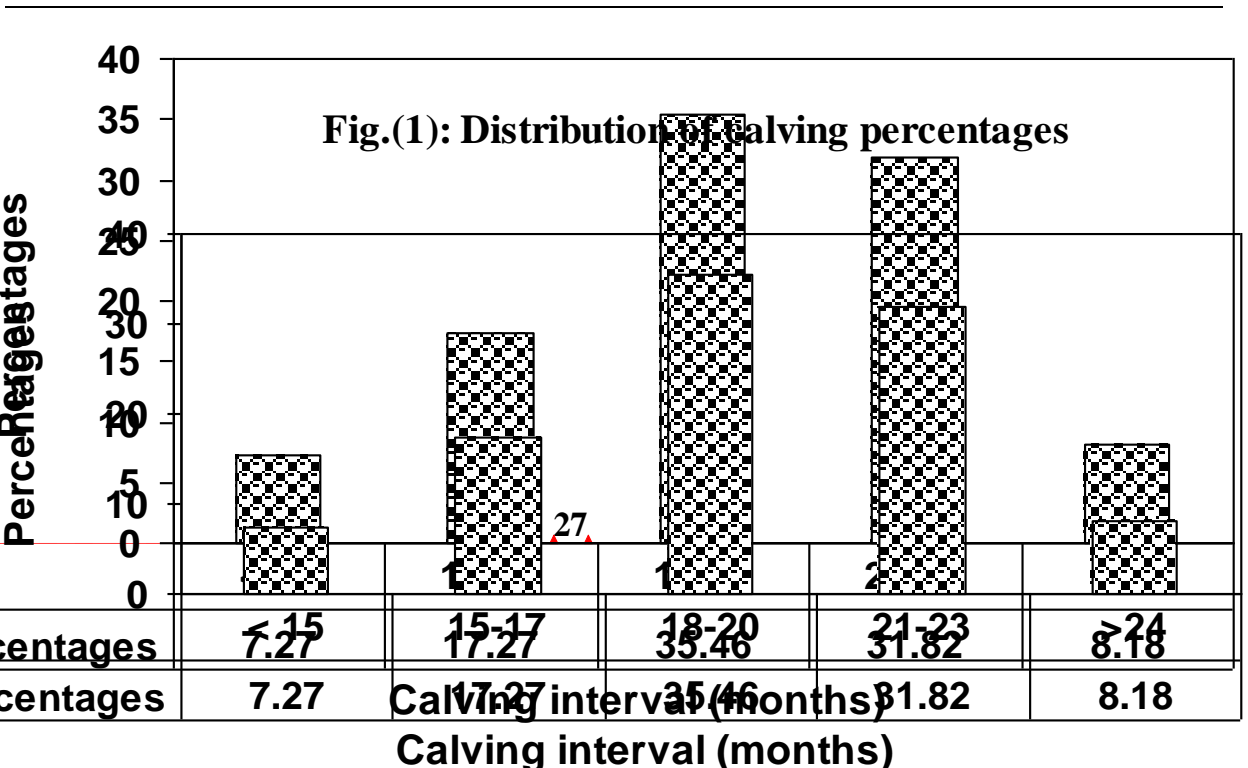




\begin{tabular}{|c|}
\hline $\begin{array}{l}\text { Formatted: Font: } 10 \mathrm{pt} \text {, Complex } \\
\text { Script Font: } 14 \mathrm{pt}\end{array}$ \\
\hline $\begin{array}{l}\text { Formatted: Justify Low, Left-to-right, } \\
\text { After: } 0 \mathrm{~cm}\end{array}$ \\
\hline Formatted: Left-to-right \\
\hline $\begin{array}{l}\text { Formatted: Justify Low, Left-to-right, } \\
\text { After: } 0 \mathrm{~cm}\end{array}$ \\
\hline $\begin{array}{l}\text { Formatted: Font: } 8 \text { pt, Bold, } \\
\text { Underline, Complex Script Font: } 8 \text { pt, } \\
\text { Not Bold, (Complex) Arabic (Egypt) }\end{array}$ \\
\hline $\begin{array}{l}\text { Formatted: Justify Low, Left-to-right, } \\
\text { After: } 0 \mathrm{~cm}\end{array}$ \\
\hline $\begin{array}{l}\text { Formatted: Font: } 10 \mathrm{pt} \text {, Complex } \\
\text { Script Font: } 10 \mathrm{pt}\end{array}$ \\
\hline $\begin{array}{l}\text { Formatted: Justify Low, Line spacing: } \\
\text { single }\end{array}$ \\
\hline Formatted: Justify Low \\
\hline
\end{tabular}

$\rightarrow$ Assiut Vet. Med. J. Vol. 58 No. 135 October 2012

A

Formatted: Font: $11 \mathrm{pt}$, Complex

Script Font: $11 \mathrm{pt}$

Formatted: Font: $10 \mathrm{pt}$, Italic,

Complex Script Font: 10 pt, Italic

Formatted: Font: 11 pt, Italic,

Complex Script Font: 11 pt, Italic

Formatted: Right-to-left, Space

Before: 0 pt, After: $0 \mathrm{pt}$

Formatted: Font: $11 \mathrm{pt}$, Italic,

Complex Script Font: 11 pt, Italic

Formatted: Font: 11 pt, Italic,

Complex Script Font: 11 pt, Italic

Formatted: Font: 11 pt, Italic,

Complex Script Font: 11 pt, Italic

Formatted: Font: $10 \mathrm{pt}$, Complex

Script Font: $10 \mathrm{pt}$

Formatted: Space Before: 0 pt, After:

0 pt

Formatted: Font: $10 \mathrm{pt}$, Complex

Script Font: $10 \mathrm{pt}$

Formatted: Justify Low, Indent: First line: $0 \mathrm{~cm}$, Space After: $0 \mathrm{pt}$, Line spacing: single

Formatted: Font: $10 \mathrm{pt}$, Complex Script Font: 10 pt

Formatted: Font: $10 \mathrm{pt}$, Complex Script Font: $10 \mathrm{pt}$

Formatted: Font: $10 \mathrm{pt}$, Complex

Script Font: $10 \mathrm{pt}$

Formatted: Font: $12 \mathrm{pt}$, Bold,

Complex Script Font: 14 pt, Bold

Formatted: Left-to-right

Formatted: Font: $12 \mathrm{pt}$, Bold,

Complex Script Font: 14 pt, Bold

تأثير سلالة الابل والعمر على الأداء التتاسلي للابل وحيدة السنام فيى السعودية

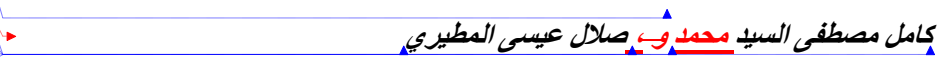

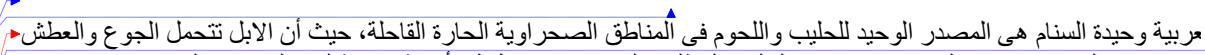

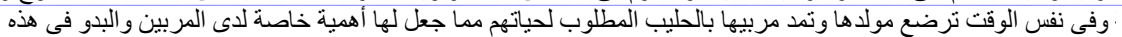

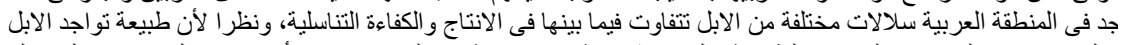

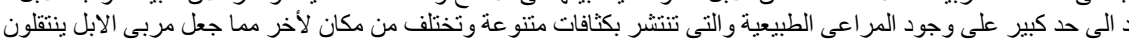

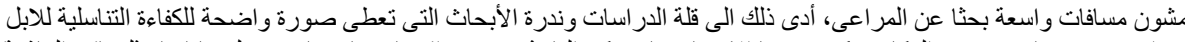

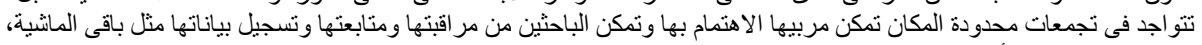

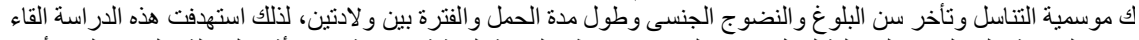

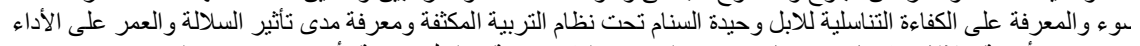

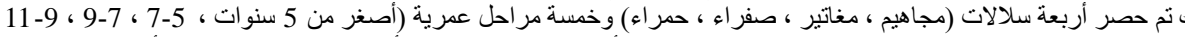

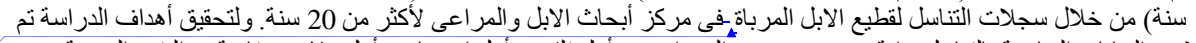

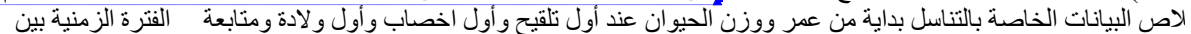

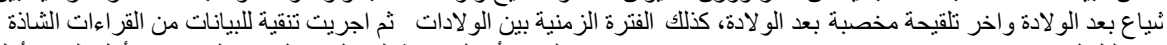

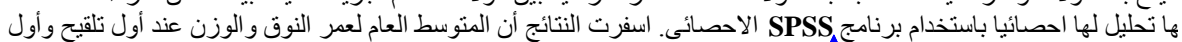

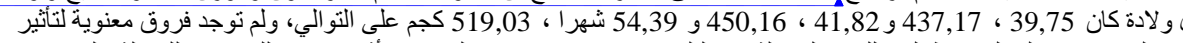

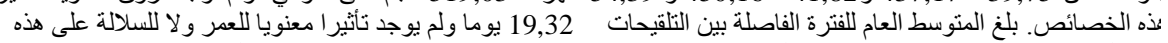

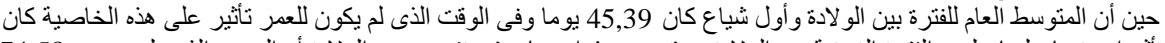

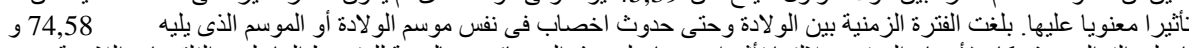

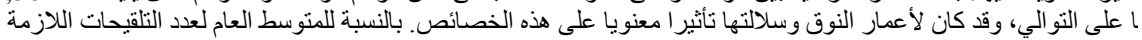

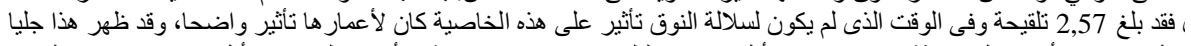

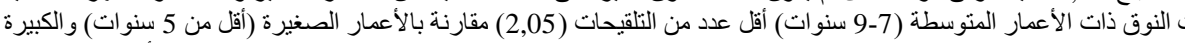

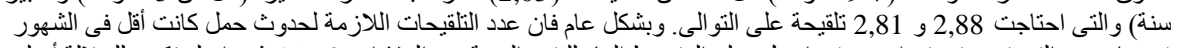

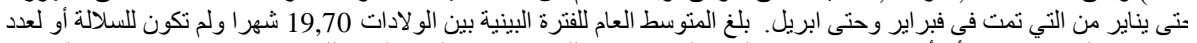

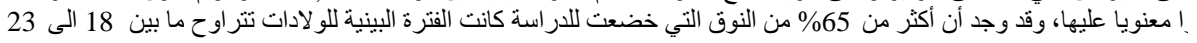


Formatted: Font: $10 \mathrm{pt}$, Complex Script Font: 14 pt

Formatted: Justify Low, Left-to-right,

After: $0 \mathrm{~cm}$

Formatted: Left-to-right

Formatted: Justify Low, Left-to-right, After: $0 \mathrm{~cm}$

Formatted: Font: $8 \mathrm{pt}$, Bold,

Underline, Complex Script Font: 8 pt,

Not Bold, (Complex) Arabic (Egypt)

Formatted: Justify Low, Left-to-right,

After: $0 \mathrm{~cm}$

$\rightarrow$ Assiut Vet. Med. J. Vol. 58 No. 135 October 2012

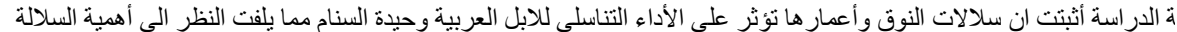

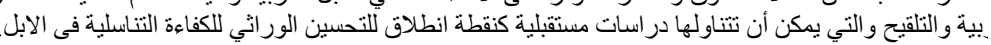

Formatted: Font: $12 \mathrm{pt}$, Bold

Complex Script Font: 14 pt, Bold

Formatted: Left-to-right

Formatted: Font: 12 pt, Bold,

Complex Script Font: 14 pt, Bold 